\title{
Core-Shell Nanostructures: Modeling, Fabrication, Properties, and Applications
}

\author{
Weihong Qi, ${ }^{1}$ Linbao Luo, ${ }^{2}$ Hai-Sheng Qian, ${ }^{3}$ Gang Ouyang, ${ }^{4}$ \\ Karuna Kar Nanda, ${ }^{5}$ and Sherine O. Obare ${ }^{6}$ \\ ${ }^{1}$ School of Materials Science and Engineering, Central South University, Changsha, Hunan 410083, China \\ ${ }^{2}$ School of Electronic Science and Applied Physics, Hefei University of Technology, Hefei, Anhui 230009, China \\ ${ }^{3}$ College of Chemistry and Life Sciences, Zhejiang Normal University, Jinhua, Zhejiang 321004, China \\ ${ }^{4}$ Key Laboratory of Low-Dimensional Quantum Structures and Quantum Control of the Ministry of Education, Department of Physics, \\ Hunan Normal University, Hunan Changsha 410081, China \\ ${ }^{5}$ Materials Research Centre, Indian Institute of Science, Bangalore 560012, India \\ ${ }^{6}$ Department of Chemistry, Western Michigan University, Kalamazoo, MI 49008, USA
}

Correspondence should be addressed to Weihong Qi, qiwh216@mail.csu.edu.cn

Received 4 December 2011; Accepted 4 December 2011

Copyright ( 2012 Weihong Qi et al. This is an open access article distributed under the Creative Commons Attribution License, which permits unrestricted use, distribution, and reproduction in any medium, provided the original work is properly cited.

Core-shell nanostructures, a family of nanomaterials, have attracted increasing research interest due to their unique structural features that consist of an inner core and an external shell of different chemical compositions. These structural features allow the possibility of combining distinctive properties of varied materials. Comparatively, core-shell nanostructures have exhibited improved physical and chemical properties relative to their single-component counterparts. The inherent emergent chemical and physical properties of core-shell nanostructures are of great importance to a potentially broader range of applications including electronics, magnetism, optics, and catalysis. So far a large number of core-shell nanostructures have been successfully fabricated using approaches ranging from laser ablation and hightemperature evaporation to carbothermal reduction and hydrothermal methods. Structural characterization of these nanostructures and determination of their unique properties for various applications have been well documented. This special issue is devoted to describing a number of unique properties and applications of core-shell nanostructures by introducing a few research papers in this field.

The paper describes a simple emulsifier-free seed emulsion polymerization technique for the synthesis of perfluoropolyether-based core-shell nanoparticles. The shell consists of relatively high Tg polystyrene and polymethylmethacrylate that have a high glass transition temperature ( $\mathrm{Tg})$ as well as polyacrylic copolymers that have a low $\mathrm{Tg}$.
The paper deals with the fabrication of polyaniline- (PANI-) $\mathrm{TiO}_{2}$ core-shell nanostructures that were prepared via a novel ionic liquid/water microemulsion method in the presence of anatase $\mathrm{TiO}_{2}$ nanoparticles. The paper reports the preparation of poly (methyl methacrylate) $/ \mathrm{SiO}_{2}\left(\mathrm{PMMA} / \mathrm{SiO}_{2}\right)$ hybrid composites using a "grafting onto" strategy, with assistance of UV light irradiation in an iron aqueous solution. The functionalization of PMMA was achieved by anchoring 3-(methacryloxy) propyl trimethoxysilane onto the nanosilica surface, followed by grafting PMMA onto the nanosilica layer with $\mathrm{FeCl}_{3}$ as a photoinitiator. The paper presents a synthesis procedure of free standing $\mathrm{Ge} / \mathrm{GeO}_{2}$ core-shell nanocrystals with tunable sizes and shell thickness. High-resolution transmission electron microscope images reveal the presence of strain in the nanocrystals and lattice distortion/dislocations in the Ge core near the interface of Ge core and $\mathrm{GeO}_{2}$ shell.

The paper of this special issue presents the preparation of silica-poly (methyl methacrylate) nanoparticles. Compared with pure natural rubber, the thermal resistance and tensile properties of such nanostructures are significantly improved. The paper is on the synchrotron X-ray absorption analysis of $\mathrm{Fe}_{2} \mathrm{O}_{3}-\mathrm{Pt}$ core-shell nanoparticles, obtained from the coreduction of iron acetylacetonate and platinum acetylacetonate. The paper reports the synthesis of a core-shell PMMAgrafted-ordered mesoporous silica by surface-initiated atom transfer radical polymerization of methyl methacrylate from 
the exterior surface of ordered mesoporous silica particle. It was found that the as-prepared OMS(ordered mesoporous silica)-g-PMMA exhibited improved properties including ionic conductivity, thermal stability, and mechanical properties.

The papersexplore the potential application of coreshell nanostructures. paper proposes a new solution-based method for the synthesis of metal-hybrid nanocomposites including $\mathrm{TiO}_{2}, \mathrm{Au} / \mathrm{TiO}_{2}, \mathrm{ZnO}$, and $\mathrm{Au} / \mathrm{ZnO}$. These semiconductor-metal hybrid composites serve as a better catalytic system for photodegradation of malathion, one of the most commonly used pesticides in developing countries. paper reports on silicon nanorods/carbon nanotubes (SiNRs/CNTs) for field emission cathodes. These novel nanostructures demonstrated improved field emission properties including a lower turn-on electric field, a lower threshold electric field, and a higher enhancement factor $B$, suggesting that such core-shell structures have good potential for field emission applications.

\author{
Weihong Qi \\ Linbao Luo \\ Hai-Sheng Qian \\ Gang Ouyang \\ Karuna Kar Nanda \\ Sherine O. Obare
}



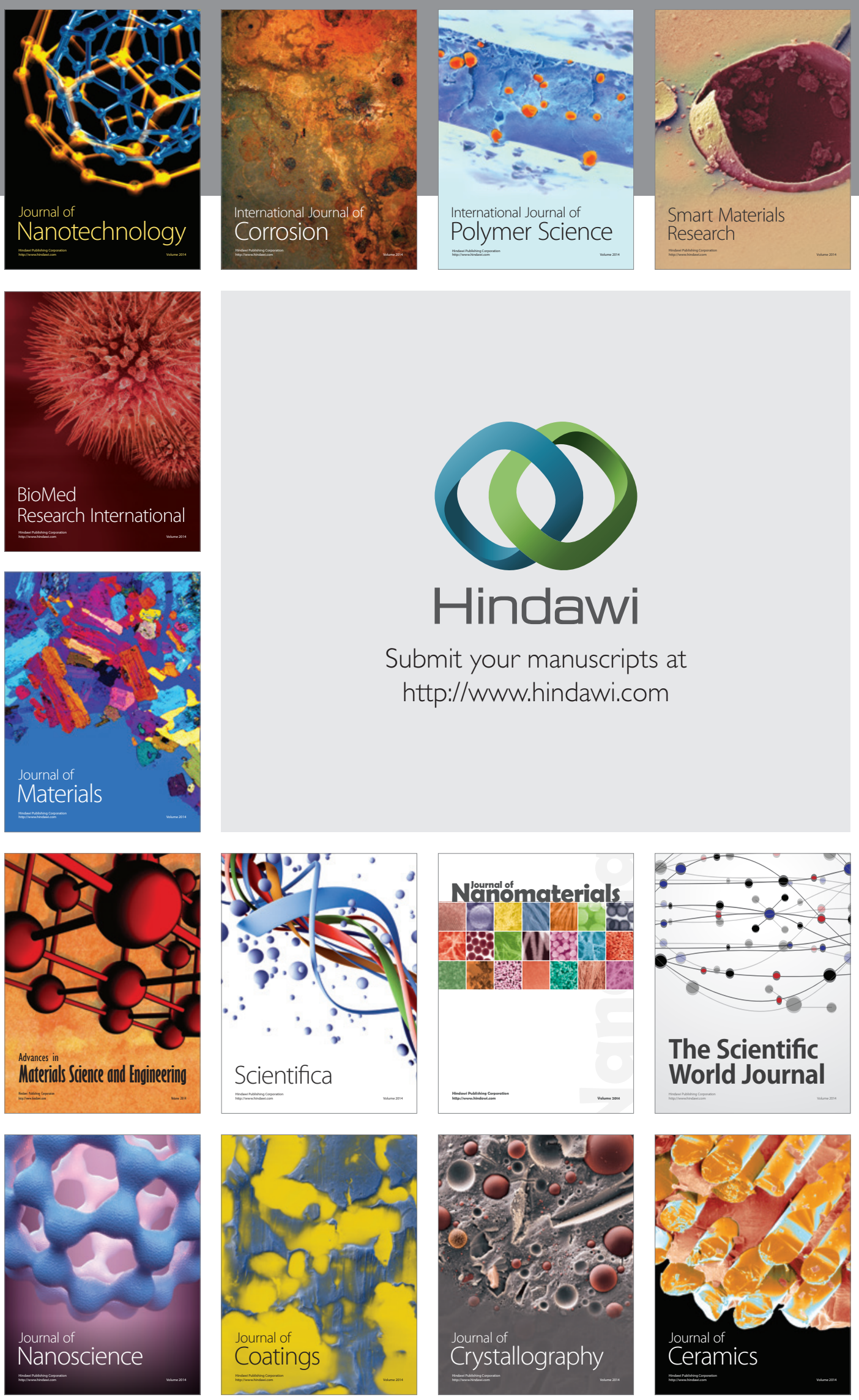

The Scientific World Journal

Submit your manuscripts at

http://www.hindawi.com

\section{World Journal}

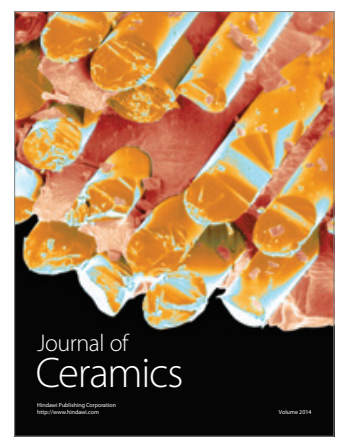


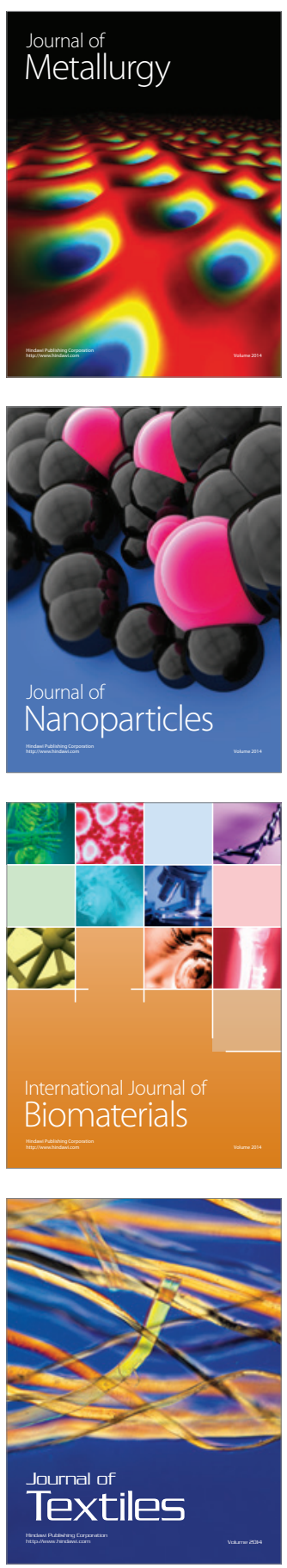